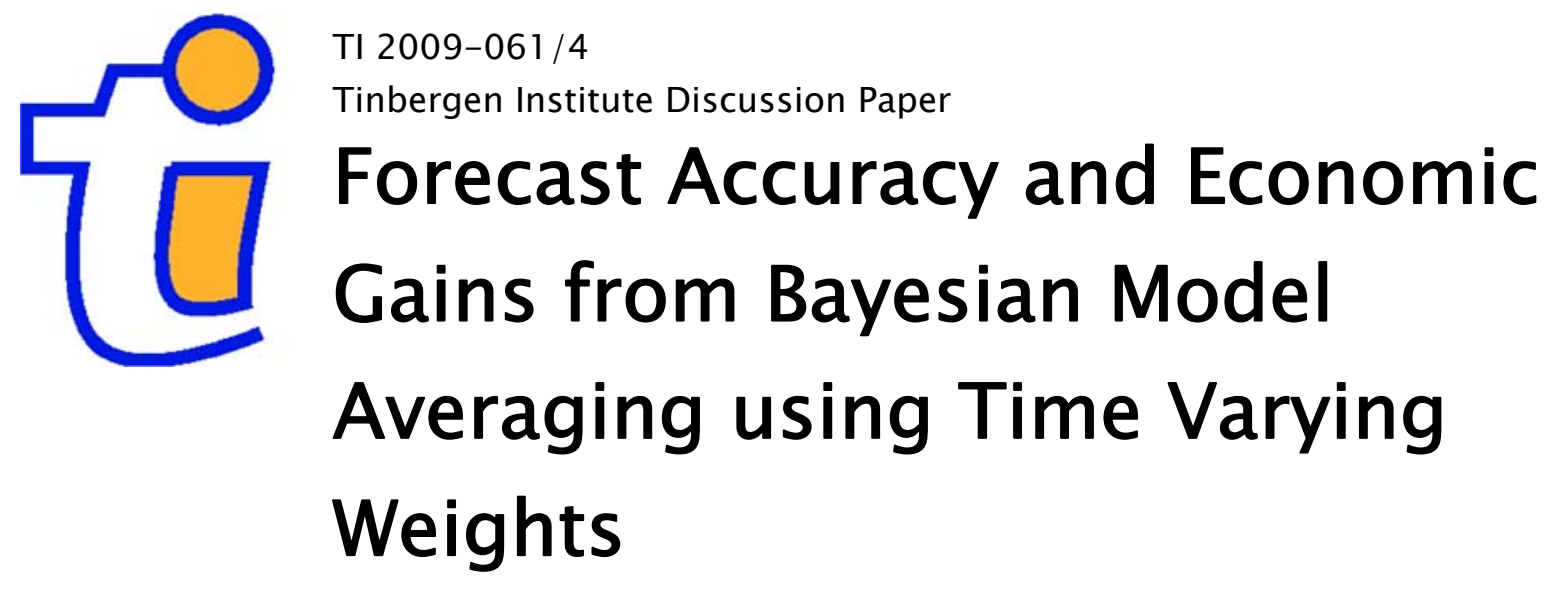

Lennart Hoogerheide'

Richard Kleijn²

Francesco Ravazzolo3

Herman K. van Dijk'

Marno Verbeek ${ }^{4}$

${ }^{\prime}$ Econometric Institute, Erasmus University Rotterdam, Tinbergen Institute;

2 PGGM, Zeist;

3 Norges Bank, Oslo;

${ }^{4}$ Rotterdam School of Management, Erasmus University Rotterdam. 


\section{Tinbergen Institute}

The Tinbergen Institute is the institute for economic research of the Erasmus Universiteit Rotterdam, Universiteit van Amsterdam, and Vrije Universiteit Amsterdam.

Tinbergen Institute Amsterdam

Roetersstraat 31

1018 WB Amsterdam

The Netherlands

Tel.: +31(0)205513500

Fax: $+31(0) 205513555$

Tinbergen Institute Rotterdam

Burg. Oudlaan 50

3062 PA Rotterdam

The Netherlands

Tel.: + $31(0) 104088900$

Fax: $+31(0) 104089031$

Most TI discussion papers can be downloaded at http://www.tinbergen.nl. 


\title{
Forecast Accuracy and Economic Gains from Bayesian Model Averaging using Time Varying Weights
}

\author{
Lennart Hoogerheide $^{1} \quad$ Richard Kleijn $^{2} \quad$ Francesco Ravazzolo $^{3}$ \\ Herman K. van Dijk ${ }^{1} \quad$ Marno Verbeek ${ }^{4}$
}

\begin{abstract}
Several Bayesian model combination schemes, including some novel approaches that simultaneously allow for parameter uncertainty, model uncertainty and robust time varying model weights, are compared in terms of forecast accuracy and economic gains using financial and macroeconomic time series. The results indicate that the proposed time varying model weight schemes outperform other combination schemes in terms of predictive and economic gains. In an empirical application using returns on the S\&P 500 index, time varying model weights provide improved forecasts with substantial economic gains in an investment strategy including transaction costs. Another empirical example refers to forecasting US economic growth over the business cycle. It suggests that time varying combination schemes may be very useful in business cycle analysis and forecasting, as these may provide an early indicator for recessions.
\end{abstract}

Key words: forecast combination, Bayesian model averaging, time varying model weights, portfolio optimization, business cycle.

\footnotetext{
${ }^{1}$ Econometric and Tinbergen Institutes, Erasmus University Rotterdam, The Netherlands ${ }^{2}$ PGGM, Zeist, The Netherlands.

${ }^{3}$ Norges Bank. Correspondence to: Francesco Ravazzolo, Norges Bank, Research Department, Bankplassen 2, 0107 Oslo, Norway. E-mail: francesco.ravazzolo@norges-bank.no

${ }^{4}$ Rotterdam School of Management, Erasmus University Rotterdam, The Netherlands
} 


\section{Introduction}

When an extensive set of forecasts of some future economic event is available, decision makers usually attempt to discover which is the best forecast, then accept this and discard the other forecasts. However, the discarded forecasts may have some independent valuable information and including them in the forecasting process may provide more accurate results. An important explanation is related to the fundamental assumption that in most cases one can not identify a priori the exact true economic process or the forecasting model that generates smaller forecast errors than its competitors. Different models may play a possibly temporary - complementary role in approximating the data generating process. In these situations, forecast combinations are viewed as a simple and effective way to obtain improvements in forecast accuracy.

Since the seminal article of Bates and Granger (1969) several papers have shown that combinations of forecasts can outperform individual forecasts in terms of loss functions. For example, Stock and Watson (2004) find that for predicting output growth in seven countries forecast combinations generally perform better than forecasts based on single models. Marcellino (2004) has extended this analysis to a large European data set with broadly the same conclusion. However, several alternative combination schemes are available and it is not clear which is the best scheme, either in a frequentist or Bayesian framework. For example, Hendry and Clements (2004) and Timmermann (2006) show that simple combinations ${ }^{1}$ often give better performance than more sophisticated approaches. Further, using a frequentist approach, Granger and Ramanathan (1984) propose the use of coefficient regression methods, Hansen (2007) introduces a Mallows' criterion, which can be minimized to select the empirical model weights, and Terui and Van Dijk (2002) generalize the least squares model weights by reformulating the linear regression model as a state space specification where the weights are assumed to follow a random walk process. Guidolin and Timmermann (2007) propose a different time varying weight combination scheme where weights have regime switching dynamics. Stock and Watson (2004) and Timmermann (2006) use the inverse mean square prediction error (MSPE) over a set of the most recent observations to compute model weights. In a Bayesian framework, Madigan and Raftery (1994) revitalize the concept of Bayesian model averaging (BMA) and apply it in an empirical application dealing with

\footnotetext{
${ }^{1}$ Simple combinations are defined as combinations with model weights that do not involve unknown parameters to be estimated; arithmetic averages constitute a simple example. Complex combinations are defined as combinations that rely on estimating weights that depend on the full variance-covariance matrix and, possibly, allow for time varying model weights.
} 
Occam's Window. Recent applications suggest its relevance for macroeconomics (Fernández, Ley, and Steel, 2001 and Sala-i-Martin, Doppelhoffer, and Miller, 2004). Strachan and Van Dijk (2008) compute impulse response paths and effects of policy measures using BMA in the context of a large set of vector autoregressive models. Geweke and Whiteman (2006) apply BMA using predictive likelihoods instead of marginal likelihoods.

This paper contributes to the research on forecast combinations by investigating several Bayesian combination schemes. We propose three schemes that allow for parameter uncertainty, model uncertainty and time varying model weights simultaneously. These approaches can be considered Bayesian extensions of the combination scheme of Terui and Van Dijk (2002).

We provide two empirical illustrations. The results indicate that time varying model weight schemes outperform other averaging schemes in terms of predictive and economic gains. The first empirical example deals with forecasting the returns on the S\&P 500 index by combining individual forecasts from four competing models. The first model assumes that a set of financial and macroeconomic variables that are related to the business cycle have explanatory power. The second model is based on the popular market saying "Sell in May and go away", also known as the "Halloween indicator", see for example Bouman and Jacobsen (2002). Low predictability of stock market return data is well documented, see for example Marquering and Verbeek (2004) and so is structural instability in this context, see for example Pesaran and Timmermann (2002) and Ravazzolo, Paap, Van Dijk, and Franses (2007). The third and fourth model are (robust) stochastic volatility models. As an investor is particularly interested in the economic value of a forecasting scheme, we test our findings in an active short-term investment exercise, with an investment horizon of one month. The forecast combination schemes with time-varying model weights provide the highest economic gains. The second empirical example refers to forecasting US economic growth over the business cycle, where we consider combinations of forecasts from six well-known time series models: an autoregressive model, two random walk models (with and without drift), an error correction model and two (robust) stochastic volatility models. It suggests that time varying weighting schemes may provide an early indicator for recessions.

The contents of this paper are organized as follows. In Section 2 we describe the different forecast combination schemes. In Section 3 we give results from an empirical application to US stock index returns which show that forecast combinations give economic gains. In Section 4 we report results from macroeconomic forecasts using US GDP growth. Section 5 concludes. 


\section{Forecast combination schemes}

Bayesian approaches have been widely used to construct forecast combinations, see for example Leamer (1978), Hodges (1987), Draper (1995), Min and Zellner (1993), and Strachan and Van Dijk (2008). In the Bayesian model averaging approach one derives the posterior density for any individual model and combines these to compute a predictive density of the event of interest. The predictive density accounts then for model uncertainty by averaging over the posterior probabilities of individual models. Since the output is a complete density, not only point forecasts but also distribution and quantile forecasts can be easily derived. We discuss four Bayesian forecast combination schemes. The first scheme is a standard approach known as Bayesian model averaging, the other three schemes obtain model weights as parameters to be estimated in linear and nonlinear regressions.

\subsection{Scheme 1: Bayesian Model Averaging (BMA)}

The predictive density of the variable $y$ at time $T+1, y_{T+1}$, given the data up to time $T$, $D_{T}$, is computed by averaging over the conditional predictive densities given the individual models with the posterior probabilities of these models as weights:

$$
p\left(y_{T+1} \mid D_{T}\right)=\sum_{i=1}^{n} p\left(y_{T+1} \mid D_{T}, m_{i}\right) P\left(m_{i} \mid D_{T}\right)
$$

where $n$ is the number of individual models; $p\left(y_{T+1} \mid D_{T}, m_{i}\right)$ is the conditional predictive density given $D_{T}$ and model $m_{i} ; P\left(m_{i} \mid D_{T}\right)$ is the posterior probability for model $m_{i}$. The conditional predictive density given $D_{T}$ and model $m_{i}$ is defined as:

$$
p\left(y_{T+1} \mid D_{T}, m_{i}\right)=\int p\left(y_{T+1} \mid D_{T}, m_{i}, \theta_{i}\right) p\left(\theta_{i} \mid D_{T}, m_{i}\right) d \theta_{i}
$$

where $p\left(y_{T+1} \mid D_{T}, m_{i}, \theta_{i}\right)$ is the conditional predictive density of $y_{T+1}$ given $D_{T}$, the model $m_{i}$ and parameters $\theta_{i} ; p\left(\theta_{i} \mid D_{T}, m_{i}\right)$ is the posterior density for parameters $\theta_{i}$ in model $m_{i}$.

The posterior probability for model $m_{i}, P\left(m_{i} \mid D_{T}\right)$, can be computed in several ways. Madigan and Raftery (1994) define it as:

$$
P\left(m_{i} \mid D_{T}\right)=\frac{p\left(y_{1: T} \mid m_{i}\right) P\left(m_{i}\right)}{\sum_{j=1}^{n} p\left(y_{1: T} \mid m_{j}\right) P\left(m_{j}\right)}
$$

where $y_{1: T}=\left\{y_{t}\right\}_{t=1}^{T} ; P\left(m_{i}\right)$ is the prior probability for model $m_{i}$; and $p\left(y_{1: T} \mid m_{i}\right)$ is the marginal likelihood for model $m_{i}$ given by:

$$
p\left(y_{1: T} \mid m_{i}\right)=\int p\left(y_{1: T} \mid \theta_{i}, m_{i}\right) p\left(\theta_{i} \mid m_{i}\right) d \theta_{i}
$$


with $p\left(\theta_{i} \mid m_{i}\right)$ the prior density for the parameters $\theta_{i}$ in model $m_{i}$. The integral in equation (4) can be evaluated analytically in the case of linear models, but not for more complex forms. Chib (1995), for example, has derived a method to compute the expression also for nonlinear examples. Laplace methods can also be used, see for example Planas, Rossi, and Fiorentini (2008). A comparative study of Monte Carlo methods for marginal likelihood evaluation, among which importance sampling and bridge sampling, is given by Ardia, Hoogerheide, and Van Dijk (2009).

Geweke and Whiteman (2006) propose a BMA scheme based on the idea that a model is as good as its predictions. The predictive density of $y_{T+1}$ conditional on $D_{T}$ has the same form as equation (1), but the posterior probability of model $m_{i}$ conditional on $D_{T}$ is now computed as:

$$
P\left(m_{i} \mid D_{T}\right)=\frac{p\left(y_{T} \mid D_{T-1}, m_{i}\right) P\left(m_{i}\right)}{\sum_{j=1}^{n} p\left(y_{T} \mid D_{T-1}, m_{j}\right) P\left(m_{j}\right)}
$$

where $p\left(y_{T} \mid D_{T-1}, m_{i}\right)$ is the predictive likelihood for model $m_{i}$, e.g. the density derived by substituting the realized value $y_{T}$ into the predictive density of $y_{T}$ conditional on $D_{T-1}$ given model $m_{i}$. Mitchell and Hall (2005) discuss the relation of the predictive likelihood to the Kullback-Leibler Information Criterion, and consequently to the frequentist combination scheme based on recursive log-score weights, see for example Kascha and Ravazzolo (2008).

We apply BMA using (5) with $p\left(y_{T} \mid D_{T-1}, m_{i}\right)$ replaced by its product over $T-k$ observations $p\left(y_{k+1} \mid D_{k}, m_{i}\right) \times \ldots \times p\left(y_{T} \mid D_{T-1}, m_{i}\right)$, where for increasing $T$ we hold constant the length $k$ of the 'initial period' of data $D_{k}$ that are only used for deriving posterior distributions. $^{2}$ That is, for forecasts of $y_{T+1}$ in later periods the predictive likelihoods and model weights are based on an expanding window of data. The densities $p\left(y_{t} \mid D_{t-1}, m_{i}\right)$ are evaluated as follows. First, parameters $\theta_{i}$ are simulated from the conditional distribution on $D_{t-1}$. Second, draws $y_{t}$ are simulated conditionally on the $\theta_{i}$ draws and $D_{t-1}$. Third, a kernel smoothing technique is used to estimate the density of $y_{t}$ in model $m_{i}$ at its realized value. The performance of alternative approaches for computing predictive likelihoods in our time varying model combination schemes is left as a topic for future research.

In all models, we specify uninformative proper priors for the parameters $\theta_{i}$. The use of predictive likelihoods rather than marginal likelihoods helps us to avoid the inference problems due to the Bartlett paradox.

\footnotetext{
${ }^{2}$ We choose $k=12$ for our applications involving monthly data.
} 


\subsection{Combination schemes using estimated regression coefficients as model weights}

The next three combination schemes estimate the weights $w_{i}$ of the models $m_{i}(i=1, \ldots, n)$ in regression form. We assume that the data $y_{t}$ satisfy the linear equation

$$
y_{t}=w_{0}+\sum_{i=1}^{n} w_{i} y_{t, i}+u_{t} \quad u_{t} \sim N\left(0, \sigma^{2}\right) \text { i.i.d. } \quad t=1,2, \ldots, T
$$

where $y_{t, i}$ has the predictive density $p\left(y_{t} \mid D_{t-1}, m_{i}\right)$ of $y_{t}$ given $D_{t-1}$ in model $m_{i}$. Clear differences with the BMA approach are that a constant term $w_{0}$ is added, and that there is no restriction that all weights must be non-negative and adding to $1 .{ }^{3}$ Therefore, the weights $w_{i}(i=1, \ldots, n)$ can not be interpreted as model probabilities. Define the model weight vector $w=\left(w_{0}, w_{1}, \ldots, w_{n}\right)^{\prime}$. We propose three novel sampling algorithms for simulating model weight vectors $w$ given the data $y_{1: T}$ and the predictive densities $p\left(y_{t} \mid D_{t-1}, m_{i}\right)(t=1, \ldots, T)$.

\section{Scheme 2: Model weights from Ordinary Least Squares in a linear model (LIN)}

A set of model weight vectors $w^{s}(s=1, \ldots, S)$ is generated by simulating independently $S$ sets of $T \times n$ draws $y_{t, i}^{s}$ from the predictive densities $p\left(y_{t} \mid D_{t-1}, m_{i}\right)(t=1, \ldots, T ; i=$ $1, \ldots, n)$, and performing an Ordinary Least Squares (OLS) regression in the model

$$
y_{t}=w_{0}+\sum_{i=1}^{n} w_{i} y_{t, i}^{s}+u_{t}^{s} \quad u_{t}^{s} \sim N\left(0, \sigma^{2}\right) \quad t=1,2, \ldots, T
$$

for each simulated set $s=1, \ldots, S$. It is well-known that in a linear model as (7) the OLS estimator $w^{s}$ is the posterior mean of $w$ under a flat prior. The generated model weights $w^{s}$ are used to combine draws $y_{T+1, i}^{s}(i=1, \ldots, n)$ from the predictive densities $p\left(y_{T+1} \mid D_{T}, m_{i}\right)$ into 'combined draws' $\tilde{y}_{T+1}^{s}$ :

$$
\tilde{y}_{T+1}^{s}=w_{0}^{s}+\sum_{i=1}^{n} w_{i}^{s} y_{T+1, i}^{s}
$$

The median of $\tilde{y}_{T+1}^{s}(s=1, \ldots, S)$ is our point forecast $\hat{y}_{T+1}$ for $y_{T+1}$, where the median is preferred over the mean because it is more robust to extreme draws. This approach can be considered as an extension of the idea of Granger and Ramanathan (1984) to combine point forecasts using weights that minimize a square loss function, to making use of Bayesian

\footnotetext{
${ }^{3}$ Granger and Ramanathan (1984) explain that the constant term must be added to avoid biased forecasts. They also conclude that this strategy is often more accurate than using restricted least squares weights.
} 
density forecasts. The model weights minimize the distance between the vector of observed values $y_{1: T}$ and the space spanned by the constant vector and the vectors of 'predicted' values $y_{1: T, i}^{s}(i=1, \ldots, n)$.

The 'combined draws' $\tilde{y}_{T+1}^{s}$ are interpreted as draws from a 'shrunk' predictive density that aims at describing the central part of the predictive density, taking into account the parameter and model uncertainty.

The assumption that the error term $u_{t}^{s}$ in (7) has constant variance $\sigma^{2}$ and no serial correlation over $t$, and has a normal distribution, is arguably violated. However, violations of this assumption have no dire consequences for the performance of the proposed point forecast $\hat{y}_{T+1}$. Roughly stated, the OLS estimator's frequentist property of consistency in combination with taking the median of a large set of 'combined draws' $\tilde{y}_{T+1}^{s}$ implies that OLS is still a usable approach. For example, the use of Generalized Least Squares (GLS) methods would not yield substantially different forecasts $\hat{y}_{T+1}$. The impact of this assumption on the 'shrunk' predictive density is arguably small; a closer look at this issue is left as a topic for further research.

\section{Scheme 3: Time-varying weights (TVW)}

The complementary roles of different models in approximating the data generating process may differ over time. Therefore, substantially better forecasts may be obtained by extending (6) to allow the model weights $w_{i}(i=1, \ldots, n)$ to change over time, resulting in

$$
y_{t}=w_{t, 0}+\sum_{i=1}^{n} w_{t, i} y_{t, i}+u_{t} \quad u_{t} \sim N\left(0, \sigma^{2}\right) \quad t=1,2, \ldots, T .
$$

Terui and Van Dijk (2002) have proposed a method that extends the linear weight combination of point forecasts to time-varying weights. We extend their approach by making use of Bayesian density forecasts, taking into account parameter uncertainty. As Terui and Van Dijk (2002) we assume that the model weights $w_{t}=\left(w_{t, 0}, w_{t, 1}, \ldots, w_{t, n}\right)^{\prime}(t=1, . ., T)$ evolve over time in the following fashion:

$$
w_{t}=w_{t-1}+\xi_{t} \quad \xi_{t} \sim N(0, \Sigma)
$$

We restrict the covariance matrix $\Sigma$ of the 'weight innovations' $\xi_{t}$ to be a diagonal matrix. The assumed independence of the weight innovations does not rule out that a posteriori there will be coinciding (large) changes of model weights. It means that this dependence is not imposed a priori. Including correlations in the weights would make the estimation 
procedure computationally more difficult, and guessing in the correlation structure can be dangerous, possibly resulting in a poor forecasting scheme. Still, we intend to analyze the extension of our scheme to non-diagonal $\Sigma$ in future research.

As in scheme 2, our algorithm results in a set of generated model weights $w_{T+1}^{s}(s=$ $1, \ldots, S)$ given the data $y_{1: T}$ and draws $y_{t, i}^{s}$ simulated from the predictive densities $p\left(y_{t} \mid D_{t-1}, m_{i}\right)$ $(t=1, \ldots, T)$. The generated model weights $w_{T+1}^{s}$ are used to transform draws $y_{T+1, i}^{s}$ $(i=1, \ldots, n)$ from the predictive densities $p\left(y_{T+1} \mid D_{T}, m_{i}\right)$ into 'combined draws' $\tilde{y}_{T+1}^{s}$ :

$$
\tilde{y}_{T+1}^{s}=w_{T+1,0}^{s}+\sum_{i=1}^{n} w_{T+1, i}^{s} y_{T+1, i}^{s}
$$

where the median of $\tilde{y}_{T+1}^{s}(s=1, \ldots, S)$ is our point forecast $\hat{y}_{T+1}$ for $y_{T+1}$. In scheme 3 , a Kalman filter algorithm (see for example Harvey (1993)) having the interpretation of a Bayesian learning approach is used to iteratively update the subsequent model weights $w_{t}^{s}$ $(t=1, \ldots, T+1)$ in the model given by

$$
y_{t}=w_{t, 0}^{s}+\sum_{i=1}^{n} w_{t, i}^{s} y_{t, i}^{s}+u_{t}^{s} \quad u_{t}^{s} \sim N\left(0, \sigma^{2}\right) \quad t=1,2, \ldots, T
$$

and (10). We fix the values of $\sigma^{2}$ and the diagonal elements of $\Sigma$. A Bayesian can interpret these assumptions as having priors on $\sigma^{2}$ and $\Sigma$ with zero variances. For each $s$ the parameters $\sigma^{2}$ and $\Sigma$ could also be estimated by maximum likelihood or MCMC methods, but we discard this to reduce computational time. ${ }^{4}$

The model weights $w_{t}^{s}$ incorporate a trade-off between minimizing the differences between the observed values $y_{1: T}$ and linear combinations of 'predicted' values $y_{1: T, i}^{s}(i=1, \ldots, n)$, and constructing a 'smooth' path of weights $w_{t}^{s}$ over time.

\section{Scheme 4: Robust time-varying weights (RTVW)}

Recently, a new specification has been developed that makes parameter estimation in case of instability over time more robust to prior assumptions, see for example Giordani and Villani (2008) and Groen, Paap, and Ravazzolo (2009) for applications. We extend the scheme 3 of time-varying model weights following the same reasoning. Then the weight innovations are

\footnotetext{
${ }^{4}$ In the financial application (with $n=4$ models) we set $\sigma^{2}$ equal to its OLS estimate in (6) allowing it to change with $s$. The $(n+1) \times 1$ vector $\operatorname{diag}(\Sigma)$ of diagonal elements of $\Sigma$ is set as $\left(0.1,0.01 \iota_{n}^{\prime}\right)^{\prime}$ with $\iota_{n}$ the $n \times 1$ vector consisting of ones, to have (small) signal-to-noise ratios in the range from 0.01 to 0.005 . For robustness we have tried different values of $\sigma^{2}$ and $\Sigma$ with signal-to-noise ratios ranging from 0.0001 to 0.1 , all resulting in qualitatively equal results. In the macroeconomic application we set $\operatorname{diag}(\Sigma)=\left(0.01,0.005 \iota_{n}^{\prime}\right)^{\prime}$.
} 
equal to the latent variables $\xi_{t, i}(i=0,1, \ldots, n)$ only with probability $\pi_{i}$ and set equal to 0 with probability $1-\pi_{i}$. That is, equation (10) becomes

$$
w_{t}=w_{t-1}+k_{t} \odot \xi_{t} \quad \xi_{t} \sim N(0, \Sigma)
$$

with $k_{t}=\left(k_{0, t}, k_{1, t}, \ldots, k_{n, t}\right)^{\prime}$, where each element $k_{i, t}$ of the vector $k_{t}$ is an unobserved $0 / 1$ variable with $P\left[k_{i, t}=1\right]=\pi_{i}$. The Hadamard product $\odot$ refers to element-by-element multiplication. $\Sigma$ is again restricted to be a diagonal matrix.

The model (12)-(13) is estimated following Gerlach, Carter, and Kohn (2000), estimating $k_{t}$ by deriving its posterior density conditional on $\sigma^{2}$ and $\Sigma$, but not on $w_{t}$. Then, we apply the Kalman Filter to estimate the latent factors $w_{t}$. We set $\sigma^{2}$ and the diagonal elements of $\Sigma$ to the same fixed values as for scheme 3 .

\section{Financial application}

In our first application we investigate the forecasting performance and economic gains obtained by applying the four forecast combination schemes to the case of US stock index returns, the continuously compounded monthly return on the S\&P 500 index in excess of the 1-month T-Bill rate, from January 1966 to December 2008, for a total of 516 observations.

We use $n=4$ individual models. The first model is based on the idea that a set of financial and macroeconomic variables contains potentially relevant factors for forecasting stock returns. Among others, Pesaran and Timmermann (1995), Cremers (2002), Marquering and Verbeek (2004) have shown that such variables can have predictive power. We include as predictors the S\&P 500 index dividend yield defined as the ratio of dividends over the previous twelve months and the current stock price, the 3-month T-Bill rate, the monthly change in the 3-month T-bill rate, the term spread defined as the difference between the 10-year T-bond rate and the 3-month T-bill rate, the credit spread defined as the difference between Moody's Baa and Aaa yields, the yield spread defined as the difference between the Federal funds rate and the 3-month T-bill rate, the annual inflation rate based on the producer price index (PPI) for finished goods, the annual growth rate of industrial production, and the annual growth rate of the monetary base measure M1. We take into account the typical publication lag of macroeconomic variables in order to avoid look-ahead bias and we include inflation, the growth rates of industrial production and the monetary base with a two-month lag. As the financial variables are promptly available, these are included with a one-month lag. We label this forecasting model "Leading indicator" (LI). 
The second forecasting model is a simple linear regression model with a constant and a dummy for November-April. It is based on the popular market saying "Sell in May and go away", also known as the "Halloween indicator" (HI) which is based on the assumption that stock returns can be predicted simply by deterministic time patterns. This suggests to buy stock in November and sell it in May. Bouman and Jacobsen (2002) show that this strategy has predictive power.

The third model allows for a well-known stylized fact on excess returns, time-varying volatility. We apply a stochastic volatility (SV) model with time varying mean:

$$
\begin{aligned}
r_{t} & =\mu_{t}+\sigma_{t} u_{t} & u_{t} \sim N(0,1) \\
\mu_{t} & =\mu_{t-1}+\xi_{1, t} & \xi_{1, t} \sim N\left(0, \tau_{1}^{2}\right) \\
\ln \left(\sigma_{t}^{2}\right) & =\ln \left(\sigma_{t-1}^{2}\right)+\xi_{2, t} & \xi_{2, t} \sim N\left(0, \tau_{2}^{2}\right)
\end{aligned}
$$

The fourth model is a robust extension of the SV model that allows for parameter instability as in Giordani and Kohn (2008). In this robust stochastic volatility (RSV) model the time-varying mean and volatility are given by

$$
\begin{array}{rlrl}
r_{t} & =\mu_{t}+\sigma_{t} u_{t} & u_{t} \sim N(0,1) & \\
\mu_{t} & =\mu_{t-1}+K_{1, t} \xi_{1, t} & \xi_{1, t} \sim N\left(0, \tau_{1}^{2}\right) \\
\ln \left(\sigma_{t}^{2}\right) & =\ln \left(\sigma_{t-1}^{2}\right)+K_{2, t} \xi_{2, t} & \xi_{2, t} \sim N\left(0, \tau_{2}^{2}\right)
\end{array}
$$

where $K_{j, t}(j=1,2 ; t=1, \ldots, T)$ is an unobserved $0 / 1$ variable with $P\left[K_{j, t}=1\right]=\pi_{j, R S V}$.

The LI and HI specifications are linear models, therefore standard Bayesian derivations apply to these, see for example Koop (2003). For estimation of the SV and RSV models we refer to Giordani, Kohn, and Van Dijk (2007).

\subsection{Evaluation}

We evaluate the statistical accuracy of the individual models and the four forecast combination schemes in terms of the root mean square error (RMSPE), and in terms of the correctly predicted percentage of sign (Sign Ratio). Moreover, as an investor is more interested in the economic value of a forecasting model than its precision, we test our conclusions in an active short-term investment exercise, with an investment horizon of one month. The investor's portfolio consists of a stock index and riskfree bonds only. At the start of each month $T+1$, the investor decides upon the fraction of her portfolio to be invested in stocks $p w_{T+1}$, based 
upon a forecast of the excess stock return $r_{T+1}$. The investor is assumed to maximize a power utility function with coefficient of relative risk aversion $\gamma$ :

$$
u\left(W_{T+1}\right)=\frac{W_{T+1}^{1-\gamma}}{1-\gamma}, \quad \gamma>1,
$$

where $W_{T+1}$ is the wealth at the end of period $T+1$, which is equal to

$$
W_{T+1}=W_{T}\left(\left(1-p w_{T+1}\right) \exp \left(r_{f, T+1}\right)+p w_{T+1} \exp \left(r_{f, T+1}+r_{T+1}\right)\right)
$$

where $W_{T}$ denotes initial wealth, and where $r_{f, T+1}$ is the riskfree rate.

Without loss of generality we set initial wealth equal to one, $W_{T}=1$, such that the investor's optimization problem is given by

$\max _{p w_{T+1}} E_{T}\left(u\left(W_{T+1}\right)\right)=\max _{p w_{T+1}} E_{T}\left(\frac{\left(\left(1-p w_{T+1}\right) \exp \left(r_{f, T+1}\right)+p w_{T+1} \exp \left(r_{f, T+1}+r_{T+1}\right)\right)^{1-\gamma}}{1-\gamma}\right)$,

where $E_{T}$ is the conditional expectation given information $D_{T}$ at time $T$. How this expectation is computed depends on how the predictive density for the excess returns is computed. If we generally denote this density as $p\left(r_{T+1} \mid D_{T}\right)$, the investor solves the following problem:

$$
\max _{p w_{T+1}} \int u\left(W_{T+1}\right) p\left(r_{T+1} \mid D_{T}\right) d r_{T+1} .
$$

The integral in (23) is approximated by generating $G$ independent draws $\left\{r_{T+1}^{g}\right\}_{g=1}^{G}$ from the predictive density $p\left(r_{T+1} \mid D_{T}\right)$, and then using a numerical optimization method to maximize the quantity:

$$
\frac{1}{G} \sum_{g=1}^{G}\left(\frac{\left(\left(1-p w_{T+1}\right) \exp \left(r_{f, T+1}\right)+p w_{T+1} \exp \left(r_{f, T+1}+r_{T+1}^{g}\right)\right)^{1-\gamma}}{1-\gamma}\right)
$$

We do not allow for short-sales or leveraging, constraining $p w_{T+1}$ to be in the $[0,1]$ interval (see Barberis (2000)).

We include eight cases in the empirical analysis below. We consider an investor who obtains a forecast of the excess stock return $r_{T+1}$ from the $n=4$ individual models (denoted LI, HI, SV and RSV) described above. Then, we consider combination forecasts using the four schemes (BMA, LIN, TVW and RTVW) from section 2, where all the individual models are combined.

We evaluate the different investment strategies by computing the ex post annualized mean portfolio return, the annualized standard deviation, the annualized Sharpe ratio and the total 
utility. Utility levels are computed by substituting the realized return of the portfolios at time $T+1$ into (20). Total utility is then obtained as the sum of $u\left(W_{T+1}\right)$ across all $T^{*}$ investment periods $T=T_{0}+1, \ldots, T_{0}+T^{*}$, where the first investment decision is made at the end of period $T_{0}$. In order to compare alternative strategies we compute the multiplication factor of wealth that would equate their average utilities. For example, suppose we compare two strategies $\mathrm{A}$ and $\mathrm{B}$. The wealth provided at time $T+1$ by the two resulting portfolios is denoted as $W_{A, T+1}$ and $W_{B, T+1}$, respectively. We then determine the value of $\Delta$ such that

$$
\sum_{T=T_{0}}^{T_{0}+T^{*}-1} u\left(W_{A, T+1}\right)=\sum_{T=T_{0}}^{T_{0}+T^{*}-1} u\left(W_{B, T+1} / \exp (\Delta)\right) .
$$

Following Fleming, Kirby, and Ostdiek (2001), we interpret $\Delta$ as the maximum performance fee the investor would be willing to pay to switch from strategy A to strategy B. For comparison of multiple investment strategies, it is useful to note that - under a power utility specification - the performance fee an investor is willing to pay to switch from strategy A to strategy B can also be computed as the difference between the performance fees of these strategies with respect to a third strategy C. ${ }^{5}$ We use this property below to infer the added value of strategies based on individual models and combination schemes by computing $\Delta$ with respect to three static benchmark strategies: holding stocks only $\left(\Delta_{s}\right)$, holding a portfolio consisting of $50 \%$ stocks and $50 \%$ bonds $\left(\Delta_{m}\right)$, and holding bonds only $\left(\Delta_{b}\right)$.

Finally, the portfolio weights in the active investment strategies change every month, and the portfolio must be rebalanced accordingly. Hence, transaction costs play a nontrivial role and should be taken into account when evaluating the relative performance of different strategies. Rebalancing the portfolio at the start of month $T+1$ means that the weight invested in stocks is changed from $p w_{T}$ to $p w_{T+1}$. We assume that transaction costs amount to a fixed percentage $c$ on each traded dollar. Setting the initial wealth $W_{T}$ equal to 1 for simplicity, transaction costs at time $T+1$ are equal to

$$
c_{T+1}=2 c\left|p w_{T+1}-p w_{T}\right|
$$

where the multiplication by 2 follows from the fact that the investor rebalances her investments in both stocks and bonds. The net excess portfolio return is then given by $r_{T+1}-c_{T+1}$. We apply a scenario with transaction costs of $0.1 \%$.

\footnotetext{
${ }^{5}$ This follows from the fact that combining (25) for the comparisons of strategies A and B with $\mathrm{C}, \sum_{T} u\left(W_{C, T+1}\right)=\sum_{T} u\left(W_{A, T+1} / \exp \left(\Delta_{A}\right)\right)$ and $\sum_{T} u\left(W_{C, T+1}\right)=\sum_{T} u\left(W_{B, T+1} / \exp \left(\Delta_{B}\right)\right)$, gives $\sum_{T} u\left(W_{A, T+1} / \exp \left(\Delta_{A}\right)\right)=\sum_{T} u\left(W_{B, T+1} / \exp \left(\Delta_{B}\right)\right)$. Using the power utility specification in (20), this can be rewritten as $\sum_{T} u\left(W_{A, T+1}\right)=\sum_{T} u\left(W_{B, T+1} / \exp \left(\Delta_{B}-\Delta_{A}\right)\right)$.
} 


\subsection{Empirical Results}

The analysis for the active investment strategies is implemented for the period from January 1987 until December 2008, involving $T^{*}=264$ one month ahead excess stock return forecasts. The individual models are estimated recursively using an expanding window of observations. The initial 12 predictions for each individual model are used as training period for combination schemes and making the first combined prediction. The investment strategies are implemented for a level of relative risk aversion of $\gamma=6 .^{6}$

Before we analyze the performance of the different portfolios, we summarize the statistical accuracy of the excess return forecasts. All the individual models give similar RMSPE statistics in Table 1, for the RSV model just the smallest and for the LI model the highest. The sign ratio is the highest for the SV model, but hardly exceeds $60 \%$, indicating low predictability. Due to this low predictability, small differences in RMSPE may have substantial economic value. We investigate this in the portfolio exercise. The SV model gives the highest Sharpe ratio, realized final utility and comparison fees $\Delta$ among the individual models. The TVW and RTVW combination schemes, however, provide much higher statistics; in particular RTVW outperforms all the other models in terms of Sharpe ratio and realized utility value, and all three $\Delta$ 's are positive. Figure 1 can help to explain these findings. Individual models allocate too low weight to the risky asset resulting in low portfolio returns. BMA has a similar problem. The LIN, TVW and RTVW combinations allocate higher weights to the stock asset, but RTVW is the only scheme that drastically reduces this weight in bear market periods as the burst of the internet bubble in 2001-2003 or the recent financial crisis in the second part of 2007 and 2008. Panel C in Table 1 shows evidence that the findings are similar when taking into account the presence of medium transaction costs.

The good performance of RTVW as compared to LIN and TVW shows that its robust flexible structure pays off. The higher portfolio weight of stock in bull markets for RTVW, as compared to the individual models and BMA, is due to the 'shrunk' predictive density. This 'shrunk' excess return distribution is not so much 'compressed' that the risky asset's portfolio weight switches from $0 \%$ to $100 \%$ when its mean changes from negative to positive values. Rather, the parameter and model uncertainty that are incorporated in this 'shrunk' predictive density imply an investment strategy with a smooth, 'moderate', yet flexible

\footnotetext{
${ }^{6}$ We also implement exercises with $\gamma=4$ and $\gamma=8$. Results are qualitatively similar and available upon request.
} 
evolvement of the risky asset's portfolio weight over time. Lettau and Van Nieuwerburgh (2008) find that the uncertainty on the size of steady-state shifts rather than their dates is responsible for the difficulty of forecasting stock returns in real time. The 'shrunk' predictive density of the RTVW scheme may be particularly informative on the current and future evolvement of this steady-state, the driving force of return predictability. This may be the explanation for the RTVW scheme's good results. We intend to analyze its performance in other portfolio management exercises in future research, in order to investigate the robustness of our findings.

\section{US real GDP Growth}

We now perform an empirical analysis on a key macroeconomic series, the U.S. real Gross Domestic Product (GDP) growth. We collected real GDP (seasonally adjusted) figures from the U.S. Department of Commerce, Bureau of Economic Analysis. The left panel of Figure 2 plots the log quarterly GDP level for our sample 1960:Q1 to 2008:Q3 (195 observations) and shows that GDP has followed an upward sloping pattern but with fluctuations around this trend. The quarterly growth rate, $\ln G D P_{t}-\ln G D P_{t-1}$, shown in the right panel of Figure 2, underlines these fluctuations with periods of positive changes followed by periods of negative changes, clearly indicating business cycles; for more details we refer to Harvey, Trimbur, and Van Dijk (2007). As in the previous section, we apply various linear and nonlinear models and forecast combinations to assess these models' suitability in a pseudo-real-time out-ofsample forecasting exercise. In the forecast exercise we use an initial in-sample period from 1960:Q1 to 1979:Q4 to obtain initial parameter estimates and we forecast the GDP growth figure for 1980:Q1. We then expand the estimation sample with the value in 1980:Q1, reestimating the parameters, and we forecast the next value for 1980:Q2. We continue this procedure up to the last value and we end up with a total of 115 forecasts.

We apply $n=6$ individual time series models to infer and forecast GDP. Four models are linear specifications, two models are time-varying parameter specifications. The first and second model are random walk models, without and with drift (RW and RWD). The third model is the autoregressive (AR) model of order 1. We follow Schotman and Van Dijk (1991) and specify a weakly informative 'regularization' prior that helps to prevent problems that could be encountered during the estimation using the Gibbs sampler, if a flat prior were used. The fourth model we apply is an error correction model (ECM). We apply the same 
model as in De Pooter, Ravazzolo, Segers, and Van Dijk (2008):

$$
\Delta y_{t}=\delta+\left(\rho_{1}+\rho_{2}-1\right)\left(y_{t-1}-\mu-\delta(t-1)\right)-\rho_{2}\left(\Delta y_{t-1}-\delta\right)+\varepsilon_{t}, \quad \varepsilon_{t} \sim N\left(0, \sigma^{2}\right)
$$

which can be rewritten as:

$$
y_{t}-\delta t=\left(1-\rho_{1}-\rho_{2}\right) \mu+\rho_{1}\left(y_{t-1}-\delta(t-1)\right)+\rho_{2}\left(y_{t-2}-\delta(t-2)\right)+\varepsilon_{t}, \quad \varepsilon_{t} \sim N\left(0, \sigma^{2}\right)
$$

The prior that we use is an extension of the prior of Schotman and Van Dijk (1991). The fifth and sixth models are a state-space model (SSM) and its robust extension (RSSM), that are given by the SV and RSV models of section 3 .

We use the root mean square prediction error (RMSPE) to compare different point forecasts. Table 2 shows that the random walk models perform poorly. For all other models, the test of Clark and West (2007) for equal forecasting quality of nested models rejects the null hypothesis versus the RW model. The AR model is a bit more precise than the ECM. The models with time varying parameters, SSM and RSSM, perform very well. Figure 3 shows that all models with fixed parameters perform poorly when GDP decreases rapidly and substantially as in NBER recessions, and it takes some quarters for models to adjust, in particular in the 2001 recession and the 2008 recession. Time-varying parameter models seem to cope better with this.

The BMA and RTVW combination schemes provide even better statistics than the SSM and RSSM models. LIN is the worst averaging scheme; LIN performs similarly to the AR and ECM models. Figure 4 shows that LIN is performing particularly poorly in the 1980's and 1990's. Weight estimates for this scheme may be highly inaccurate as the number of individual models is relatively large and instability possibly high. Moreover, Figure 4 indicates that the other averaging schemes react much faster to sharp decreases in GDP. Especially the RTVW scheme may early indicate recessions: before both the 1991 and 2001 crises its point forecast decreases substantially with approximately $0.5 \%$.

To sum up, our results suggest that model averaging may be very beneficial in business cycle analysis and forecasting. The combination method must, however, be chosen carefully and it should cope with estimation efficiency and structural instability, in particular if weights are estimated in regression equations. Again, more extensive studies should be performed to investigate the robustness of our findings, for example over different countries and periods. 


\section{Final remarks}

The empirical applications have indicated, firstly, that averaging strategies can give higher predictive quality than selecting the best model; secondly, that properly specified time varying model weights yield higher forecast accuracy and substantial economic gains compared with other averaging schemes. The presented results lead to multiple directions for future research. As we already mentioned, interesting possibilities for further research are a rigorous analysis of the impact of some assumptions - both on theoretical aspects and practical applications - and an extensive study on the robustness of our findings.

Another topic for further research is to compare our results to other time varying weight combination schemes, such as regime switching, see e.g. Guidolin and Timmermann (2007), or schemes that carefully model breaks, see e.g. Ravazzolo, Paap, Van Dijk, and Franses (2007). For the application to portfolio management, a natural extension is the prediction of multivariate returns processes. The proposed combination schemes can also be adapted to the specific prediction of variance, skewness or kurtosis.

\section{Acknowledgements}

This paper is a substantial revision and extension of Ravazzolo, Van Dijk, and Verbeek (2007). We are very grateful to participants of the Conference of the 50-th Anniversary of the Econometric Institute 2006, and the Conference on Computational Economics and Finance, Geneva, 2007, for their helpful comments on earlier versions of the paper. Herman K. van Dijk gratefully acknowledges the financial assistance from the Netherlands Organization of Research (under grant \# 400-07-703). The views expressed in this paper are our own and do not necessarily reflect the views of Norges Bank (the Central Bank of Norway). Any remaining errors or shortcomings are the authors' responsibility. 


\section{References}

Ardia, D., Hoogerheide, L. F., and Van Dijk, H. K. 2009. To Bridge, to Warp or to Wrap? A comparative study of Monte Carlo methods for efficient evaluation of marginal likelihoods. Tinbergen institute report 09-017/4.

Barberis, N. 2000. Investing for the Long Run When Returns Are Predictable. Journal of Finance 55: 225-264.

Bates, J. M., and Granger, C. W. J. 1969. Combination of Forecasts. Operational Research Quarterly 20: 451-468.

Bouman, S., and Jacobsen, B. 2002. The Halloween Indicator, 'Sell in May and Go Away': Another Puzzle. American Economic Review 92 (5): 1618-1635.

Chib, S. 1995. Marginal Likelihood from the Gibbs Output. Journal of American Statistical Association 90: 972-985.

Clark, T. E., and West, K. D. 2007. Approximately normal tests for equal predictive accuracy in nested models. Journal of Econometrics 138 (May): 291-311.

Cremers, K. J. M. 2002. Stock Return Predictability: A Bayesian Model Selection Perspective. Review of Financial Studies 15: 1223-1249.

De Pooter, M., Ravazzolo, F., Segers, R., and Van Dijk, H. K. 2008. Bayesian Near-Boundary Analysis in Basci Macroeconomic Time-Series Models. Advances in Econometrics 23: 331402.

Draper, D. 1995. Assessment and Propagation of Model Uncertainty. Journal of the Royal Statistical Society Series B 56: 45-98.

Fernández, C., Ley, E., and Steel, M. F. J. 2001. Model uncertainty in cross-country growth regressions. Journal of Applied Econometrics 16: 563-576.

Fleming, J., Kirby, C., and Ostdiek, B. 2001. The Economic Value of Volatility Timing. Journal of Finance 56: 329-352.

Gerlach, R., Carter, C., and Kohn, R. 2000. Efficient Bayesian Inference for Dynamic Mixture Models. Journal of the American Statistical Association 95: 819-828. 
Geweke, J., and Whiteman, C. 2006. Bayesian Forecasting. In G. Elliot, C. Granger, and A. Timmermann(ed.) Handbook of Economic Forecasting North-Holland.

Giordani, P., and Kohn, R. 2008. Efficient Bayesian Inference for Multiple Change-Point and Mixture Innovation Models. Journal of Business 85 Economic Statistics 26: 66-77.

Giordani, P., Kohn, R., and Van Dijk, D. 2007. A Unified Approach to Nonlinearity, Outliers and Structural Breaks. Journal of Econometrics 137: 112-137.

Giordani, P., and Villani, M. 2008. Forecasting macroeconomic time series with locally adaptive signal extraction. Working Paper.

Granger, C. W. J., and Ramanathan, R. 1984. Improved Methods of Combining Forecasts. Journal of Forecasting 3: 197-204.

Groen, J., Paap, R., and Ravazzolo, F. 2009. Real-Time Inflation Forecasting in a Changing World. Working paper.

Guidolin, M., and Timmermann, A. 2007. Forecasts of US Short-term Interest Rates: A Flexible Forecast Combination Approach. forthcoming in Journal of Econometrics.

Hansen, B. E. 2007. Least Squares Model Averaging. Econometrica 75(4): 1175-1189.

Harvey, A. C. 1993. Time Series Models: . Pearson Education.

Harvey, A. C., Trimbur, T. M., and Van Dijk, H. K. 2007. Bayes Estimates of the Cyclical Component in Twentieth Century U.S. GrossDomestic Product. In G. L. Mazzi, and G. Savio(ed.) Growth and Cycle in the Eurozone Palgrave MacMillan, New York.

Hendry, D. F., and Clements, M. P. 2004. Pooling of Forecasts. Econometric Reviews 122: $47-79$.

Hodges, J. 1987. Uncertainty, Policy Analysis and Statistics. Statistical Science 2: 259-291.

Kascha, C., and Ravazzolo, F. 2008. Combining Inflation Density Forecasts. Norges Bank working paper 2008-22.

Koop, G. 2003. Bayesian Econometrics. West Sussex, England: John Wiley \& Sons Ltd.

Leamer, E. 1978. Specification Searches: . New York: Wiley. 
Lettau, M., and Van Nieuwerburgh, S. 2008. Reconciling the Return Predictability Evidence. The Review of Financial Studies 21: 1607-1652.

Madigan, D., and Raftery, A. 1994. Model Selection and Accounting for Model Uncertainty in Graphical Models Using Occam's Window. Journal of the American Statistical Association 89: $1335-1346$.

Marcellino, M. 2004. Forecasting Pooling for Short Time Series of Macroeconomic Variables. Oxford Bulletin of Economic and Statistics 66: 91-112.

Marquering, W., and Verbeek, M. 2004. The Economic Value of Predicting Stock Index Returns and Volatility. Journal of Financial and Quantitative Analysis 39 (2): 407-429.

Min, C., and Zellner, A. 1993. Bayesian and Non-Bayesian Methods for Combining Models and Forecasts with Applications to Forecasting International Growth Rates. Journal of Econometrics 56: 89-118.

Mitchell, J., and Hall, S. G. 2005. Evaluating, comparing and combining density forecasts using the KLIC with an application to the Bank of England and NIESER "fan" charts of inflation.. Oxford Bulletin of Economics and Statistics 67: 995-1033.

Pesaran, M. H., and Timmermann, A. 1995. Predictability of Stock Returns: Robustness and Economic Significance. Journal of Finance 50: 1201-1228.

Pesaran, M. H., and Timmermann, A. 2002. Market Timing and Return Predictability Under Model Instability. Journal of Empirical Finance 9: 495-510.

Planas, C., Rossi, A., and Fiorentini, G. 2008. The marginal likelihood of Structural Time Series Models, with application to the euroarea and US NAIRU. Working Paper Series 21-08, Rimini Centre for Economic Analysis.

Ravazzolo, F., Paap, R., Van Dijk, D., and Franses, P. H. 2007. Bayesian Model Averaging in the Presence of Structural Breaks. In M. Wohar, and D. Rapach(ed.) Forecasting in the Presence of Structural Breaks and Model Uncertainty Elsevier.

Ravazzolo, F., Van Dijk, H. K., and Verbeek, M. 2007. Predictive gains from forecast combinations using time varying model weights. Econometric institute report 2007-26. 
Sala-i-Martin, X., Doppelhoffer, G., and Miller, R. 2004. Determinants of long-term growth: A Bayesian averaging of classical estimates (BACE) approach. American Economic Review 94: 813-835.

Schotman, P., and Van Dijk, H. K. 1991. A Bayesian Analysis of the Unit Root in Real Exchange Rates. Journal of Econometrics 49: 195-238.

Stock, J. H., and Watson, M. 2004. Combination Forecasts of Output Growth in a Sevencountry Data Set. Journal of Forecasting 23: 405-430.

Strachan, R., and Van Dijk, H. K. 2008. Bayesian Averaging over Many Dynamic Model Structures with Evidence on the Great Ratios and Liquidity Trap Risk. Tinbergen Institute report 2008-096/4, Erasmus University Rotterdam.

Terui, N., and Van Dijk, H. K. 2002. Predictability in the Shape of the Term Structure of Interest Rates. International Journal of Forecasting 18: 421-438.

Timmermann, A. 2006. Forecast Combinations. In G. Elliot, C. W. J. Granger, and A. Timmermann(ed.) Handbook of Economic Forecasting North-Holland. 


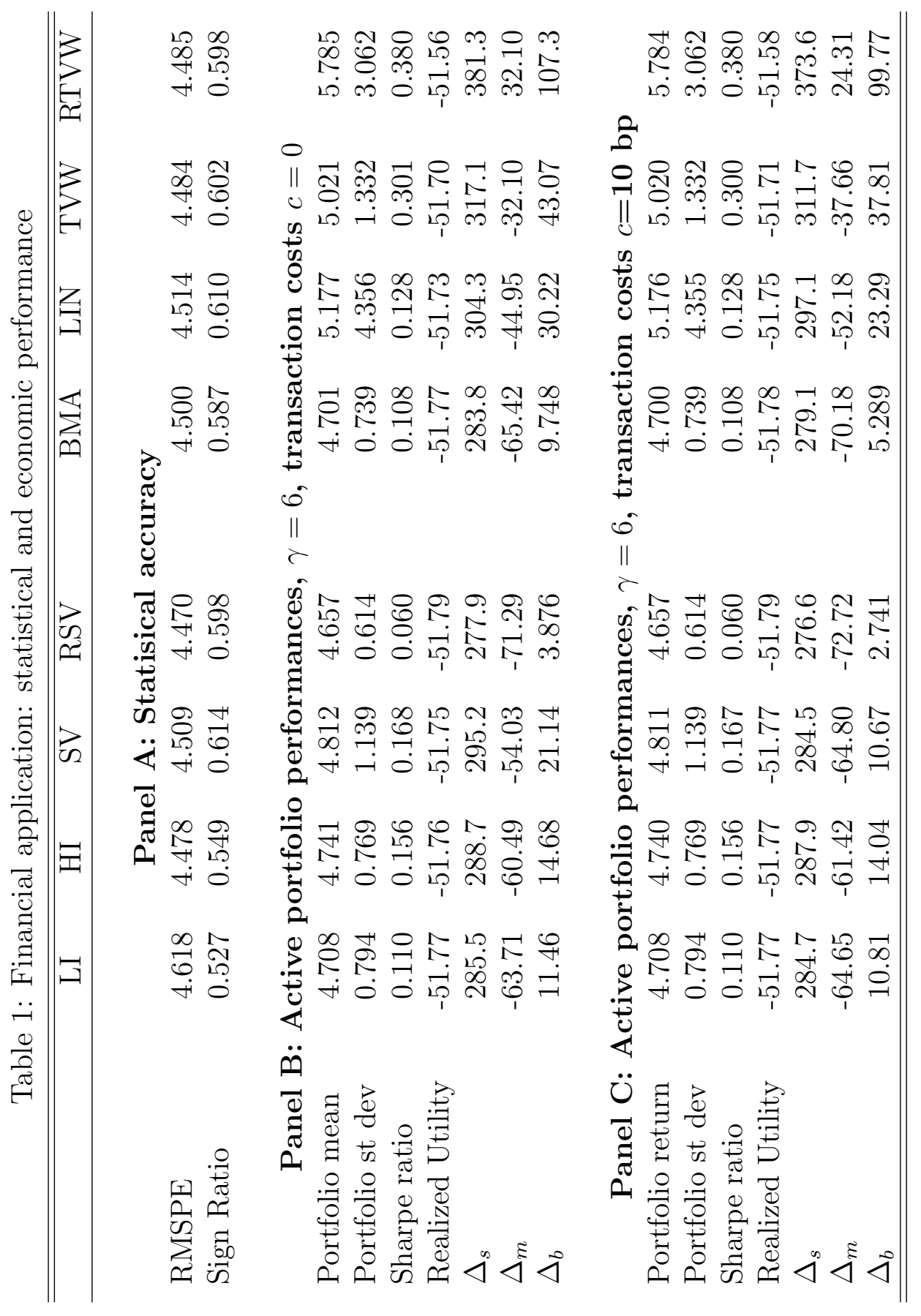


Figure 1: Financial application: portfolio weight of stock (S\&P500)
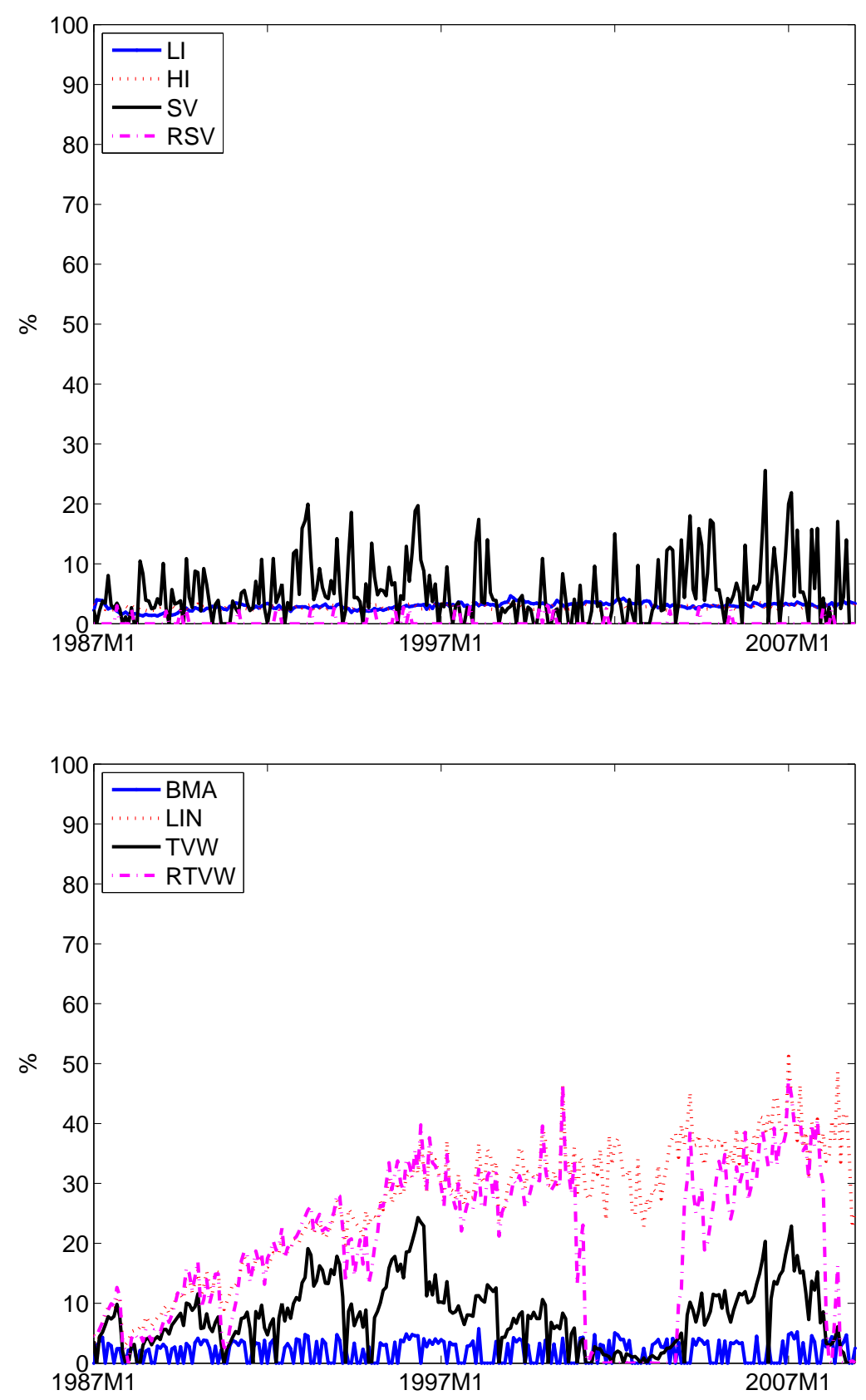

Note: The graphs show the portfolio weight on the risky asset (S\&P500) over the out-of-sample period associated to active asset management given by individual models in the left panel and combination schemes in the right panel. 
Table 2: Forecasting U.S. real GDP growth (in \% terms): root mean square prediction error (RMSPE)

\begin{tabular}{lcll}
\hline \hline \multicolumn{2}{l}{ individual models } & \multicolumn{2}{c}{ combinations } \\
\hline RW & 1.650 & BMA & 0.718 \\
RWD & 0.863 & LIN & 0.829 \\
AR & 0.772 & TVW & 0.757 \\
ECM & 0.790 & RTVW & 0.727 \\
SSM & 0.730 & & \\
RSSM & 0.747 & & \\
\hline \hline
\end{tabular}


Figure 2: US real GDP
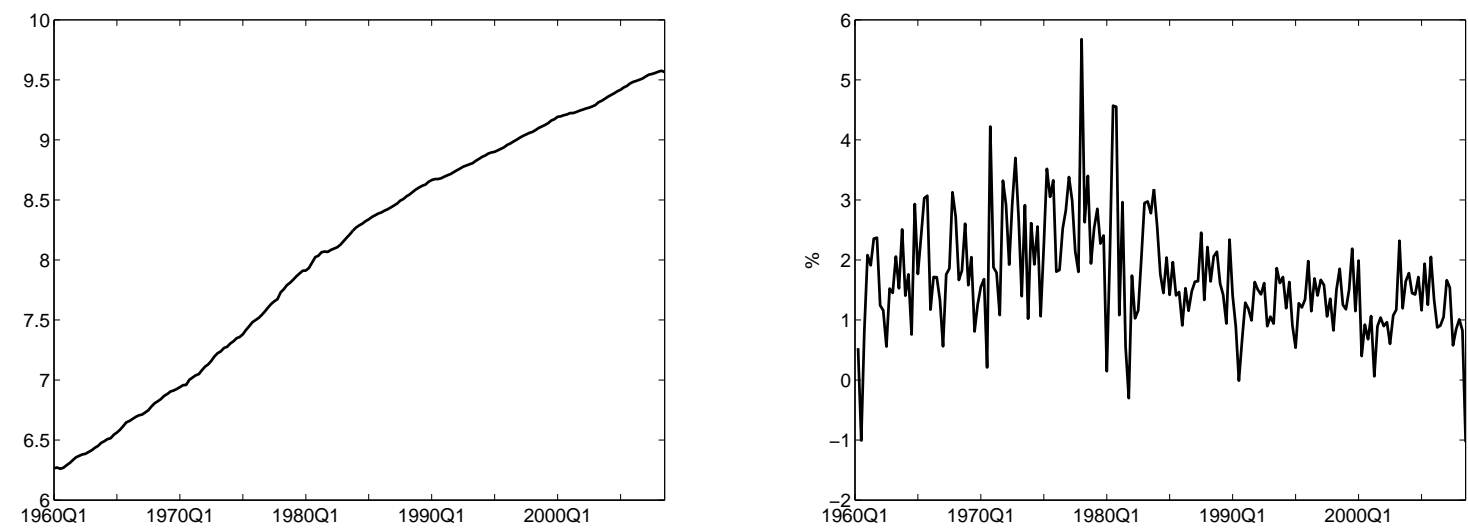

Note: Quarterly log levels of U.S. real GDP (left) and quarterly GDP growth rate in $\%$ terms (right). The sample is 1960:Q1 - 2008:Q3. 
Figure 3: US real GDP: point forecasts
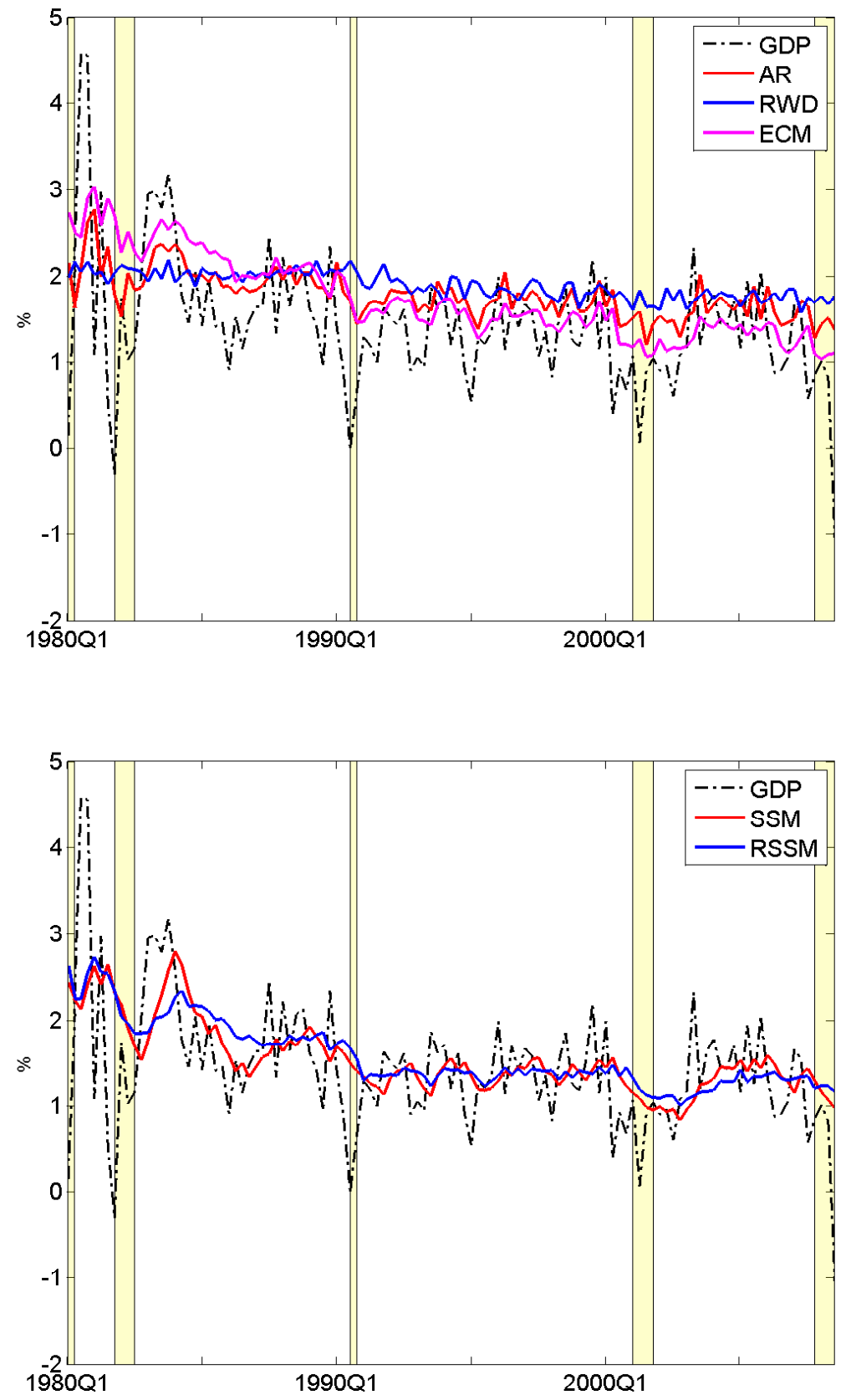

Note: Quarterly U.S. real GDP growth (in \%) and point forecasts given by individual models. Vertical bars highlight NBER recession periods. 
Figure 4: US real GDP: point forecasts

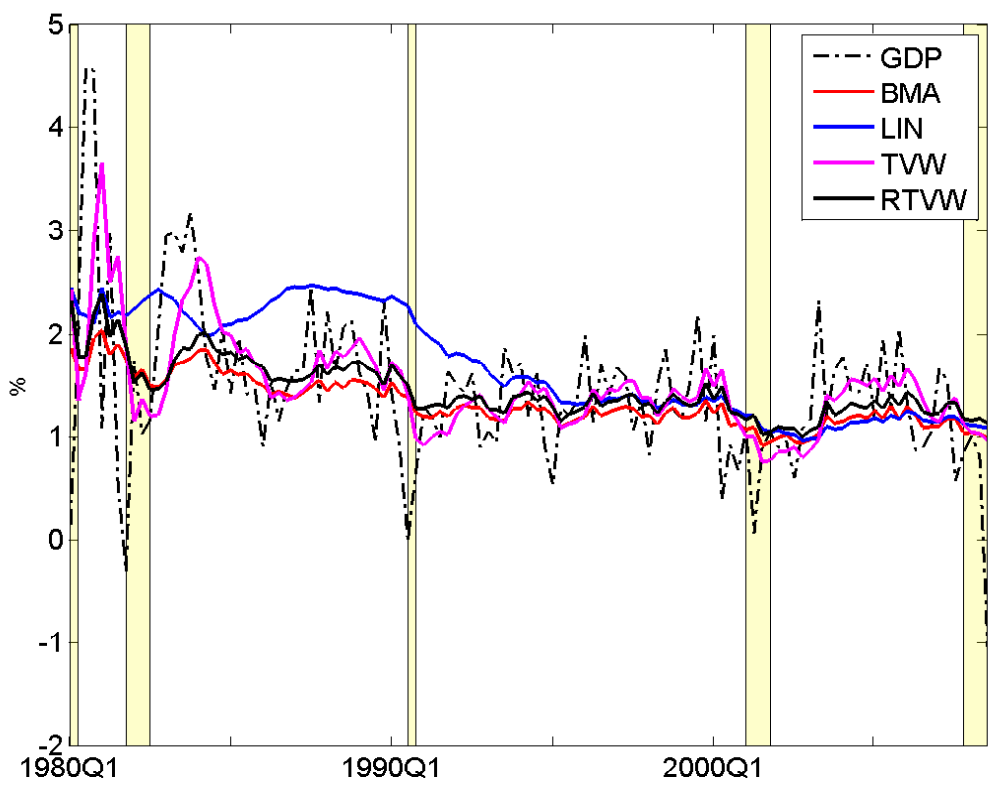

Note: Quarterly U.S. real GDP growth (in \%) and point forecasts given by combination schemes. Vertical bars highlight NBER recession periods. 